\title{
Excisão cirúrgica de um osteocondroma de raiz nervosa da cauda equina de cão
}

\author{
Surgical Excision of an Osteochondroma in a Nerve Root of the Cauda Equina of a Dog
}

\author{
Juliana Campos de Barros' ${ }^{1}$ Larissa Garbelini Valentim ${ }^{1}$, Raira Costa Dias' ${ }^{1}$ Alfredo Hajime Tanaka Pereira', \\ Giovana Wingeter Di Santis ${ }^{2}$ \& Mônica Vicky Bahr Arias³
}

\begin{abstract}
Background: Spinal neoplasms are classified into extradural, intradural/extramedullary or intramedullary. Intradural/extramedullary tumors include meningiomas and nerve sheath tumors, which arise from meninges or peripheral nerves around the spinal cord. Clinical signs are related to dysfunction of the involved nerve and include pain, nerve root signature and atrophy. Osteochondromas are benign tumors located within the bone, on its surface or in extra-osseous regions, when they are classified as soft tissue osteochondoma. The aim of this study is to describe a case of an osteochondroma in a nervous root of the cauda equina in a dog, whose surgical resection allowed the resolution of the clinical signs.

Case: A 12-year-old, male, Labrador Retriever dog, was presented with a 40-day history of progressive, painful, pelvic limb paresis, with no improvement when treated with analgesics and acupuncture. Neurological abnormalities included paraparesis, sometimes worse in the left pelvic limb, that was carried flexed at the level of the stifle, hindlimb atrophy, decreased interdigital reflexes and pain in the lumbar spinal region, mainly over L6 vertebra. Results of blood count and serum biochemical analysis were unremarkable. Computed tomography (CT) of the lumbosacral area was performed and the evaluation of images in transverse and reconstructed dorsal and sagittal planes allowed the visualization of a hyperattenuating and calcified round structure with $8 \mathrm{~mm} \times 6 \mathrm{~mm}$, in the left side of vertebral canal, at the level of caudal epiphysis of L6. Lumbosacral (L7-S1) CT abnormalities, as subchondral sclerosis, mild disc margin bulging, spondylosis deformans and foraminal proliferation were also observed but were considered clinically insignificant. Then, a dorsal L6 laminectomy was performed and after opening of the vertebral canal it was observed the presence of an ovoid mass, in the left side of the medullary cone, measuring approximately $0.9 \mathrm{~cm}$ in length, originating from a sacral nerve root. This structure was excised and submitted to histopathological evaluation. The dog improved after surgery without any complications, and neurological functions were gradually recovered. The histopathology of the mass was confirmed as an osteochondroma. Discussion: The history and neurologic examination findings as well the improvement after surgery were compatible with the lesion visualized by TC in L6, rather than the degenerative changes in L7-S1. Regarding the position of the mass, the main differential would be nerve sheath tumor, one of the most common types of primary neoplasia of the peripheral nervous system, however in a CT study in dogs with brachial plexus and nerve roots neoplasms, calcification of the tumor was not observed. Most of benign cartilaginous tumors of soft tissue are chondromas/osteochondromas, and this lesion should not be confused with multiple osteochondroma or multiple cartilaginous exostoses, which have been reported as multifocal, proliferative lesions involving the surface of ribs, vertebrae and pelvis of young dogs. If this lesion is unique, it is called osteochondroma. There are two reports of chondromas into the first lumbar vertebrae of dogs, with adherence to the floor of the vertebral canal and one report of an osteochondroma originated from the left pedicle of T11 in a cat. Radiculopathy and myelopathy caused by osteochondromas inside the spine are rare in humans and description of this tumor arising from nerve roots were not found in the veterinary literature. Probably the origin of the mass was metaplasia of the meninges surrounding the nervous root. In conclusion, neurological examination, CT and surgical exploration allowed the diagnosis and removal of a soft tissue osteochondroma, a rare benign tumor, with good patient recovery.
\end{abstract}

Keywords: diseases, nervous system, neoplasms, spinal nerve roots.

Descritores: doenças, sistema nervoso, neoplasias, raízes nervosas. 


\section{INTRODUÇÃO}

Dentre as afecções neurológicas da coluna vertebral, medula espinhal e raízes nervosas, neoplasias devem ser consideradas no rol de diagnósticos diferenciais, principalmente em cães de grande porte, idosos, com hiperpatia e outras alterações neurológicas [2,16,20].

As neoplasias que afetam a coluna vertebral, medula espinhal ou raízes nervosas são classificadas de acordo com sua localização anatômica em extradurais, intradural/extramedular e intramedulares e de acordo com a origem em primários ou secundários [2]. As neoplasias intradurais/extramedulares em geral são primárias e tem origem da bainha de mielina das raízes nervosas ou meninges [2]. Neoplasias benignas na raiz nervosa e localizadas dentro do canal medular, cuja origem não seja da bainha de mielina, são raras em cães [21].

Os tumores derivados da cartilagem e que afetam a coluna vertebral são a exostose cartilaginosa, osteocondroma, condroma e condrossarcoma [18]. Osteocondromas são projeções ósseas, envolvidas por cartilagem e localizados na superfície externa dos ossos, que podem se apresentar como lesão única (osteocondroma solitário) ou múltipla (osteocondromas múltiplos/exostoses cartilaginosas) [10]. Acometem ossos chatos e longos de animais e seres humanos jovens, mas raramente o interior do canal medular [7,8]. Quando isso acontece, a neoformação originada das estruturas ósseas se projeta para o interior do canal medular.

Como a metaplasia cartilaginosa da meninge no interior do canal vertebral formando um osteocondroma é rara [4], o objetivo deste relato é descrever esta neoplasia em raiz nervosa da cauda equina de um cão, com resolução dos sinais clínicos e retorno das funções neurológicas após excisão cirúrgica.

\section{CASO}

Um cão, macho, da raça Labrador, de 37 $\mathrm{kg}$ e 12 anos de idade foi encaminhado ao Hospital Veterinário da Universidade Estadual de Londrina (HV-UEL) com histórico de dificuldade de locomoção progressiva com os membros pélvicos, com evolução de 40 dias. Estava sendo tratado com analgésicos, anti-inflamatórios e acupuntura, porém sem melhora. Ao exame clínico os parâmetros fisiológicos estavam dentro dos limites normais de referência. No exame neurológico, os membros torácicos encontravam-se normais, porém observou-se paresia, atrofia muscular e flacidez dos músculos caudais da coxa, com claudicação à impotência funcional, que alternava entre os membros posteriores, mas era mais acentuada no membro posterior esquerdo, que se mantinha flexionado. Havia ainda diminuição do reflexo interdigital e aumento do reflexo patelar bilateralmente, que foi classificado como pseudo-hiperrreflexia do patelar. $\mathrm{O}$ paciente não elevava ou movia a cauda e à palpação da coluna havia dor em região lombar, principalmente sobre a vértebra L6 (Figura 1A). A nocicepção dos membros estava preservada e o reflexo cutâneo do tronco encontrava-se normal. Com base nesses achados, a lesão foi localizada neuroanatomicamente como síndrome lombossacra (L4-S3) e os principais diagnósticos diferenciais foram doenças inflamatórias, infecciosas, degenerativas e/ou neoplásicas. O exame radiográfico da coluna LS não apresentou anormalidades, bem como os exames laboratoriais (hemograma completo e bioquímica sérica). Foi proposta a realização de tomografia computadorizada (TC) da região lombar. O paciente foi medicado com $4 \mathrm{mg}$ $\mathrm{kg}^{-1}$ cloridrato de tramadol $\left(\operatorname{Tramal}^{\circledR}\right)^{1}$ a cada $8 \mathrm{~h}$ pela via oral e $10 \mathrm{mg} \mathrm{kg}^{-1}$ de gabapentina (Neurontin $\left.{ }^{\circledR}\right)^{1}$, a cada $8 \mathrm{~h}$ pela via oral, até que os resultados de exames estivessem disponíveis para a escolha do tratamento definitivo, sem melhora do quadro.

$\mathrm{Na}$ TC foi visibilizado opacificação do canal vertebral de L6-7 e L7-S1 obliterando a gordura epidural, degeneração da face caudal do corpo vertebral de L7, discreto material hiperatenuante no aspecto ventral do canal vertebral ao nível de L4-5 e L5-6 e presença de uma estrutura mineralizada no aspecto dorsolateral esquerdo do cone medular da cauda equina, na altura da epífise caudal de L6, de aproximadamente $8 \mathrm{~mm}$ de comprimento por $4 \mathrm{~mm}$ de largura (Figura 2). Após avaliação dos exames de imagem, suspeitou-se que a estrutura mineralizada era a responsável pelos sinais neurológicos e optou-se pela realização de cirurgia exploratória.

Foi realizada tricotomia ampla da região lombar e antissepsia prévia com clorexidine degermante $(\text { Riohex } 2 \%)^{2}$. O paciente foi tranquilizado com 0,3 $\mathrm{mg} \mathrm{kg}^{-1} \mathrm{de}$ midazolam (Dormonid $\left.{ }^{\circledR}\right)^{3}$ pela via intravenosa, a anestesia foi induzida com $4 \mathrm{mg} \mathrm{kg}^{-1} \mathrm{em}$ bôlus de propofol (Propovan $\left.{ }^{\circledR}\right)^{4}$ intravenoso e mantida com isoflurano (Isoflurano $\left.{ }^{\circledR}\right)^{5}$ em oxigênio. Cefazolina $\left(\mathrm{Kefazol}^{\circledR}\right)^{6}$ na dose de $30 \mathrm{mg} \mathrm{kg}^{-1}$ foi administrada pela via intravenosa profilaticamente $30 \mathrm{~min}$ antes 
da cirurgia e ao final da mesma e mantida durante as primeiras $24 \mathrm{~h}$ do período pós-operatório, sendo administrada a cada 8 h. Após antissepsia cirúrgica e delimitação do local a ser operado com panos de campo, a vértebra L6 foi abordada dorsolateralmente por meio de uma incisão mediana dorsal desde a altura do processo espinho da L5 até o processo espinhoso de S1. Procedeu-se a divulsão da musculatura epaxial bilateralmente com afastador de periósteo, que foi mantida desviada com afastador de Gelpi autoestático, para exposição do processo espinhoso e lâmina dorsal das vértebras L6 e L7. Com auxílio de uma goiva, o processo espinhoso de L6 foi excisado e em seguida, com uma broca cilíndrica conectada à um micromotor elétrico cirúrgico à velocidade de $35000 \mathrm{rpm}$, foi realizado o desgaste da camada cortical externa e medular da lâmina dorsal de L6. A excisão da camada cortical interna foi finalizada com pinça de Lowe-Kerrinson. Após exploração cirúrgica observou-se a presença de uma massa ovóide, firme e esbranquiçada, medindo aproximadamente $0,9 \mathrm{~cm}$ de comprimento por $0,4 \mathrm{~cm}$ de largura, originando-se de uma raiz nervosa do lado esquerdo do cone medular, provavelmente sacral, não sendo possível separar a massa da raiz, assim a mesma foi excisada por secção da raiz, cranial e caudalmente à massa, com tesoura delicada. O material foi encaminhado para exame histopatológico. Em seguida, foi realizada a lavagem do sítio cirúrgico com solução salina estéril e procedeu-se à síntese de rotina da fáscia muscular, subcutâneo e pele.

Após a cirurgia, os sinais de dor foram avaliados por meio do exame físico e a analgesia pós-operatória foi assegurada com infusão contínua de sulfato de morfina $\left(\text { Dimorf }^{\circledR}\right)^{4}$ na dose de $5 \mathrm{mg} \mathrm{kg-1}$ por hora pela via intravenosa e diazepan $\left(\text { Diazepam }^{\circledR}\right)^{7}$ na dose de $0,3 \mathrm{mg} \mathrm{kg}^{-1}$, pela via intravenosa a cada $8 \mathrm{~h}$, durante um dia, seguido por cloridrato de tramadol na dose de $3 \mathrm{mg} \mathrm{kg}^{-1}$ a cada $8 \mathrm{~h}$ por uma semana. Observou-se melhora gradativa e apreciável do quadro neurológico e após uma semana o paciente já caminhava melhor. Ao exame neurológico permanecia com leve fraqueza, porém a dor estava controlada e não havia mais paresia ou impotência funcional dos membros pélvicos. Após um mês houve melhora evidente da locomoção e da função da cauda (Figura 1B). Em contato telefônico após 3 e 6 meses, o paciente não apresentou recidiva dos sinais neurológicos.
No exame histopatológico observou-se que a massa era composta por tecido cartilaginoso hialino e estroma fibroso com presença de condrócitos típicos e áreas de tecido conjuntivo fibrovascular hipocelular. O citoplasma dos condrócitos era moderadamente basofílico e o núcleo redondo a ovoide, de cromatina vesicular e nucléolo evidente. Observou- se também ilhas de osteoide e focos de calcificação com formação de medula óssea, assim a neoplasia foi classificada como ostecondroma e devido a sua localização e provável origem da meninge da raiz nervosa como osteocondroma de tecidos moles (Figura 3).

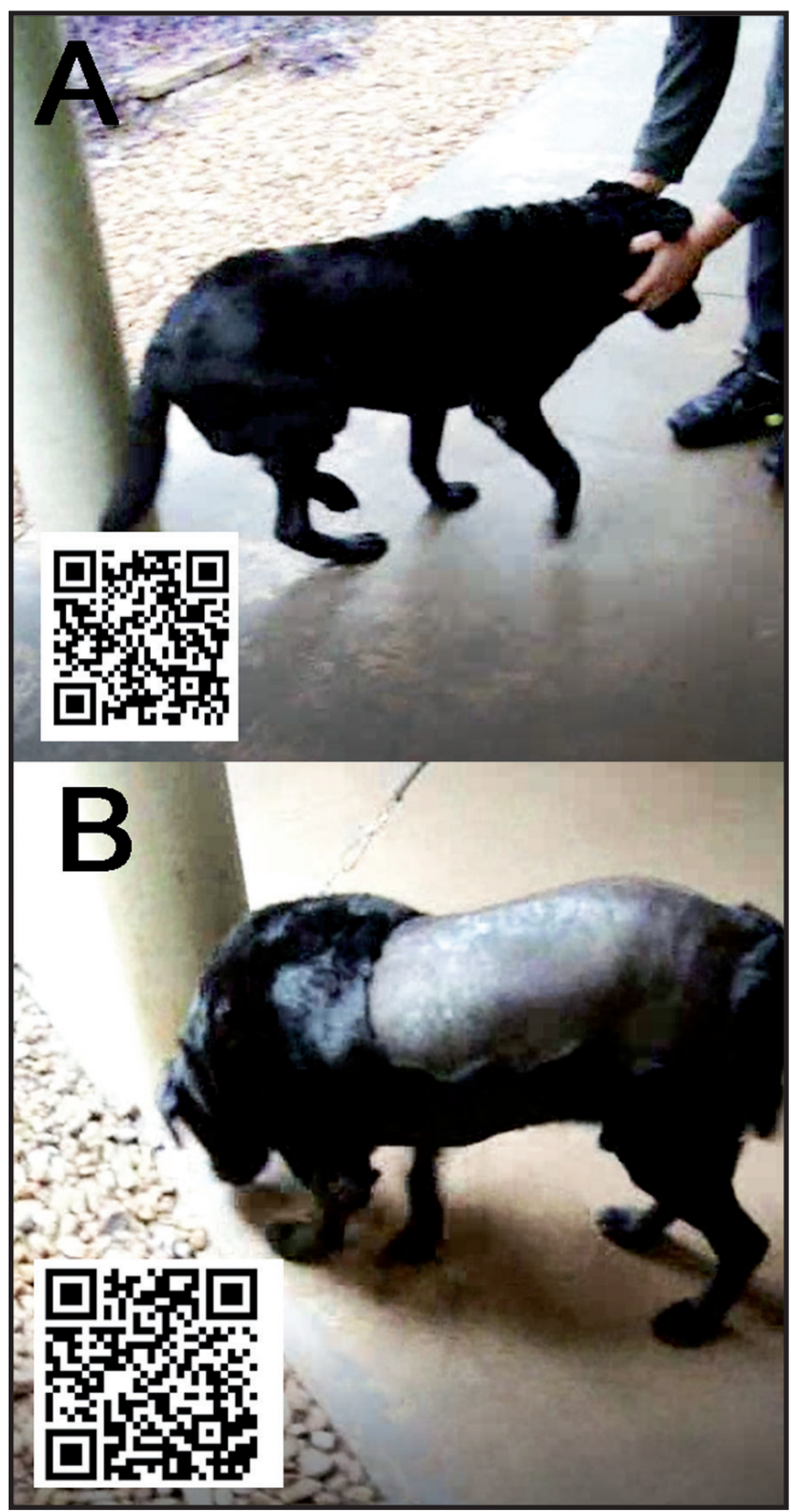

Figura 1. Imagem fotográfica do paciente canino, raça Labrador, de $37 \mathrm{~kg}$ e 12 anos de idade. A- Antes do procedimento cirúrgico. B- três semanas após o procedimento cirúrgico de excisão de um osteocondroma do interior do canal vertebral de L6. O escaneamento do QR Code com aplicativo de celular "QR code reader" irá mostrar um vídeo com a locomoção do paciente nestes momentos. 


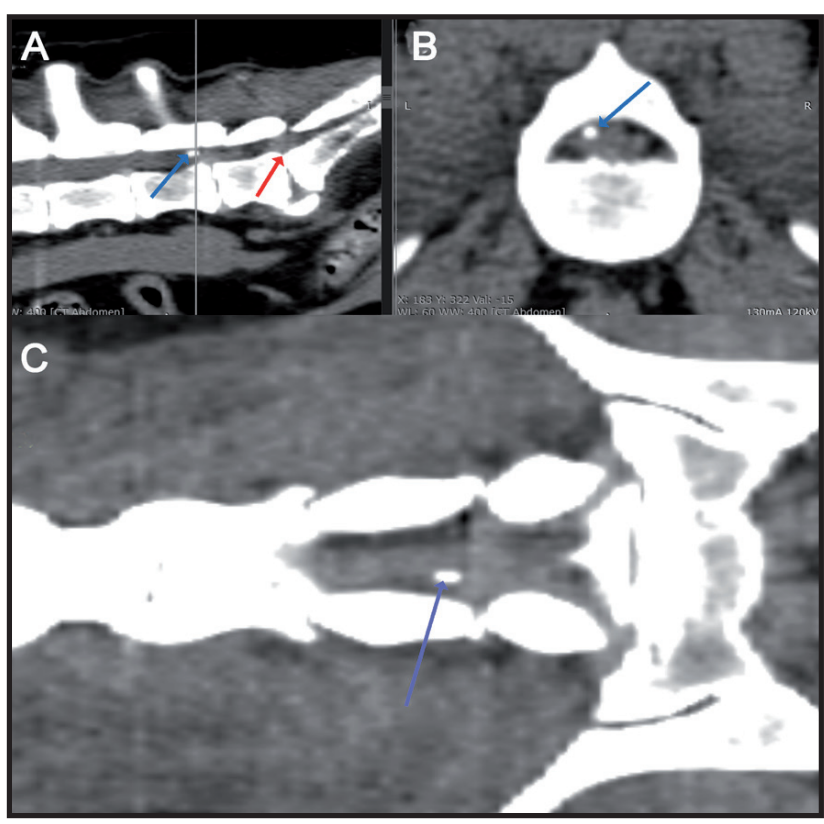

Figura 2. Imagem fotográfica de tomografia computadorizada da coluna lombossacra do paciente canino, raça Labrador, de $37 \mathrm{~kg}$ e 12 anos de idade. A- Tomografia em corte sagital; a seta azul aponta para a estrutura cilíndrica e calcificada no interior do canal vertebral, na altura da epífise caudal de L6. A linha perpendicular está na altura do corte transversal mostrada em B. A seta vermelha aponta para a articulação lombosacra que apresentava alterações degenerativas. B- Tomografia em corte transversal, na altura da epífise caudal de L6. A seta azul aponta para a estrutura cilíndrica e calcificada, no interior do canal vertebral do lado esquerdo. C- Tomografia em corte dorsal, na mesma altura de A e B, a seta azul aponta para a estrutura diagnosticada como osteocondroma.

\section{DISCUSSÃO}

As causas mais comuns de síndrome lombossacra crônica, progressiva e com dor em cães são estenose lombossacra degenerativa (ELD), doença do disco intervertebral, neoplasias e doenças inflamatórias [15]. Embora no exame de imagem do paciente houvesse alterações degenerativas entre L7-S1, é importante lembrar que este achado é comum nos exames de imagem de cães idosos de grande porte [1]. A avaliação dos sinais clínicos sugeriu síndrome da cauda equina por ELD [15], mas os aspectos que reforçaram a suspeita de que a estrutura calcificada visibilizada na TC [12] fosse responsável pelo quadro clínico foram a presença de dor mais acentuada na palpação da vértebra L6, o quadro clínico progressivo, o fato do animal manter os membros pélvicos flexionados, principalmente o esquerdo, indicando sinal de raiz [2,3] e a idade avançada do paciente. Há uma descrição de quadro clínico semelhante em um cão com um lipoma perirradicular fusiforme no interior do canal da vértebra L6 [21].

As exostoses cartilaginosas múltiplas ou osteocondromatose ou osteocondroma, são lesões proliferativas benignas, multifocais, envolvidas por

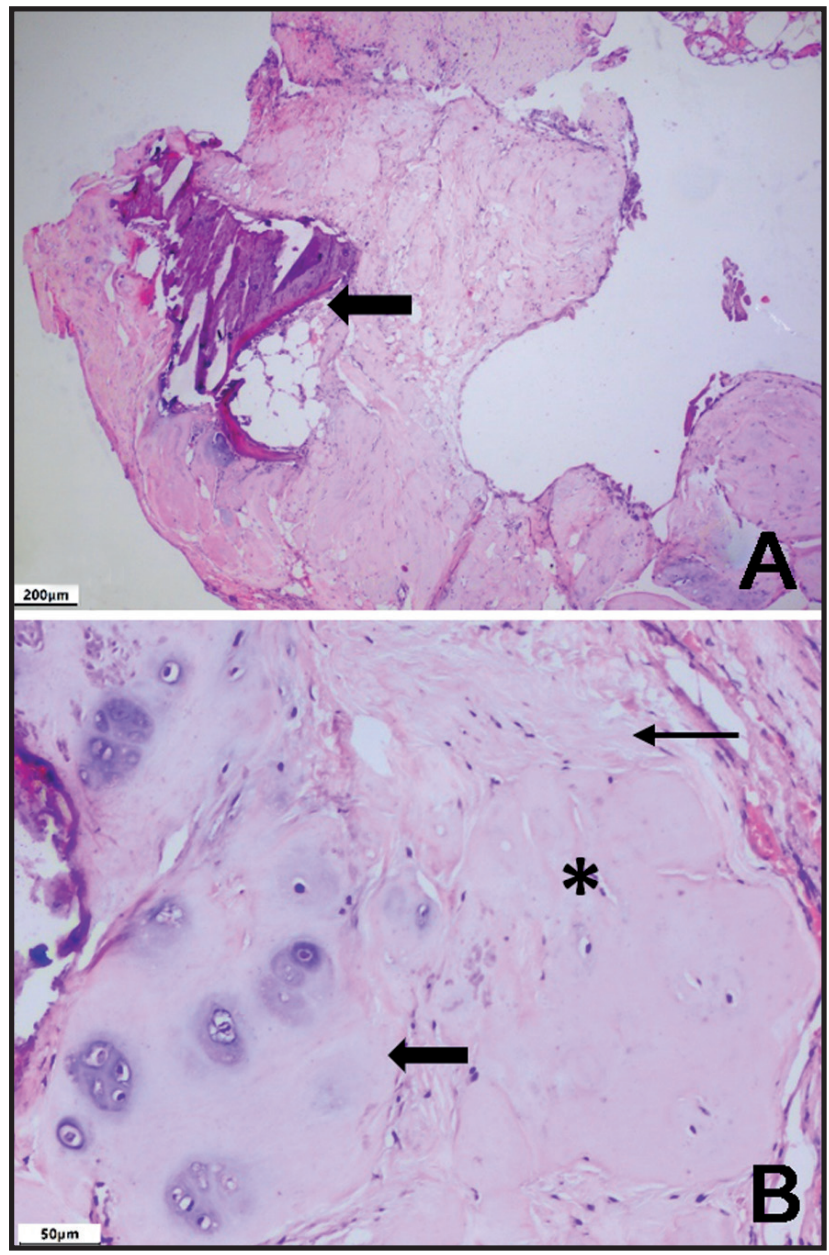

Figura 3. Microfotografia digital. Neoformação ressectada de raiz da cauda equina de um cão, coloração Hematoxilina e Eosina (HE). A- Amostra constituída predominantemente de tecido cartilaginoso hialino, além de pequenos focos de ossificação com formação de medula óssea (seta) e áreas de tecido conjuntivo fibrovascular hipocelular. B- Transição (*) entre o tecido conjuntivo (seta fina) e o cartilaginoso (seta espessa), com aquisição de progressiva basofilia (tom arroxeado) resultante da produção de matriz condroide e organização dos ninhos de condrócitos. [A: 4x ; B: 20x].

uma capa cartilaginosa, que se elevam na superfície externa das escápulas, costelas, vertebras e pelve de cães jovens apresentando continuidade com o osso do qual se originaram $[2,6,8,10]$, o mesmo ocorrendo em humanos, equinos, cães e gatos jovens com osteocondroma solitário da coluna $[11,17]$. Ocasionalmente a massa, originada do tecido ósseo da vértebra, cresce em direção ao interior do canal vertebral, comprimindo o tecido nervoso $[11,12]$, mas isso não foi observado no presente relato.

O osteocondroma apresenta similaridade com condroma, um tumor benigno raro, caracterizado pela formação de cartilagem, que também podem se originar de qualquer parte da vértebra, como processos espinhoso e transverso, lâmina, pedículo e corpo vertebral [9], mas que não tem o componente tecidual ósseo [8]. Há dois relatos em que o condroma estava localizado nas 
primeiras vértebras lombares, com aderência ao assoalho do canal vertebral $[13,14]$ e um caso de condroma originado da cartilagem da faceta articular C4-C5 [18]. Os dois tipos de neoformação descritos acima são diferentes do osteocondroma encontrado na raiz nervosa do cão do presente relato, que pode ser classificado como osteocondroma de tecidos moles, uma neoplasia cartilaginosa benigna que não tem origem em tecido ósseo ou cartilaginoso e pode originar-se da dura-máter entre outros tecidos [5,8]. Há uma descrição de condroma de tecidos moles em uma mulher de meia idade, originado da dura-máter no interior do canal vertebral lombar na altura da vértebra L2, com diagnóstico realizado por ressonância magnética e com bons resultados após excisão cirúrgica [5], mas não foram encontrados relatos de osteocondromas radiculares em humanos ou cães. A neoplasia provavelmente originou-se por metaplasia da camada aracnoide [5] da meninge que envolve a raiz nervosa [7].

Em relação à localização da massa, o principal diferencial seria tumor da bainha nervosa, um dos tipos mais comuns de neoplasia primária do sistema nervoso periférico, que pode se originar das células de Schwann, tecido conectivo peri e endoneural, axônios ou corpo celular do neurônio, mas que são mais comuns no plexo braquial [2]. Além disso, em um estudo tomográfico em 24 cães com neoplasias no plexo braquial e raízes nervosas, não foi observado calcificação das massas [19]. Entretanto, neoplasias no sistema nervoso periférico podem ter a aparência tubular ou esférica, como visto no presente relato [2].

O tratamento recomendado para a maioria das neoplasias epidurais e intradurais/extramedulares é a ressecção cirúrgica, cujos objetivos são descomprimir o tecido nervoso, estabelecer o diagnóstico histopatológico e preservar função a neurológica [2,3]. A exploração cirúrgica permitiu a excisão da massa, que tinha origem da raiz nervosa lateralmente ao cone medular e não estava aderida à lâmina vertebral interna, faceta articular ou assoalho vertebral, ao contrário dos casos de condroma e osteocondroma vertebral descritos em cães [13,14,18] ou seres humanos [9]. A excisão da raiz não comprometeu a função neurológica, havendo melhora do quadro, provavelmente por diminuição da compressão do cone medular. Em um cão com sinais neurológicos semelhantes ao do presente relato e com diagnóstico tomográfico da presença de uma massa hipoatenuante na mesma região, um lipoma periradicular foi excisado, junto com fascículos de uma raiz nervosa, com boa evolução do quadro após o procedimento cirúrgico [21].

Em conclusão, o exame neurológico associado à tomografia computadorizada e exploração cirúrgica permitiu o diagnóstico e excisão de um osteocondroma de tecidos moles de raiz nervosa em cão, cuja ocorrência é extremamente rara, havendo boa recuperação do paciente.

\section{MANUFACTURERS}

${ }^{1}$ Laboratórios Pfizer Ltda. Guarulhos, SP, Brazil.

${ }^{2}$ Rioquímica S.A. São José do Rio Preto, SP, Brazil.

${ }^{3}$ Roche Diagnóstica Brasil. São Paulo, SP, Brazil.

${ }^{4}$ Laboratório Cristália. São Paulo, SP, Brazil.

${ }^{5}$ Instituto BioChimico Indústria Farmacêutica Ltda. Rio de Janeiro, RJ, Brazil.

${ }^{6}$ ABL. Antibióticos do Brasil. São Paulo, SP, Brazil.

${ }^{7}$ União Química Farmacêutica Nacional S.A. São Paulo, SP, Brazil.

Declaration of interest. The authors report no conflicts of interest. The authors alone are responsible for the content and writing of the paper.

\section{REFERENCES}

1 Amort K.H., Ondreka N., Rudorf H., Stock K.F., Distl O., Tellhelm B., Kramer M. \& Wigger A. 2012. MR-imaging of lumbosacral intervertebral disc degeneration in clinically sound German Shepherd dogs compared to other breeds. Veterinary Radiology \& Ultrasound. 53(3): 289-295.

2 Bagley R.S. 2010. Spinal neoplasms in small animals. The Veterinary Clinics of North America. Small Animal Practice. 40(5): 915-927.

3 Besalti O., Caliskan M., Can P., Vural S.A., Algin O. \& Ahlat O. 2016. Imaging and surgical outcomes of spinal tumors in $18 \mathrm{dogs}$ and one cat. Journal of Veterinary Science. 17(2): 225-234.

4 Braund K.G. 2003. Neoplasia of the Nervous System. In: Braund K.G. (Ed). Clinical Neurology in Small Animals. Localization, Diagnosis and Treatment. Ithaca: IVIS. [Fonte:<http://www.ivis.org/special_books/Braund/braund26/ chapter_frm.asp?LA=1>]. [Accessed online in December 2017].

5 Cho H.J., Shim J.C., Kim H.K., Suh J.H., Lee G.J., Kim Y.H., Seo J.G. \& Lee H.K. 2009. A soft tissue chondroma originating from the dura mater of the lumbar spinal canal and it mimicked a nerve sheath tumor: a case report with the MR imaging. Journal of the Korean Society of Radiology. 60(4): 221-225. 
6 Engel S., Randall E.K., Cuddon P.A., Webb B.T. \& Aboellail T.A. 2014. Imaging diagnosis: Multiple cartilaginous exostoses and calcinosis circumscripta occurring simultaneously in the cervical spine of a dog. Veterinary Radiology \& Ultrasound. 55(3): 305-309.

7 Evans H.E. \& De Lahunta A. 2013. Miller's Anatomy of the Dog. 4th edn. St. Louis: Saunders-Elsevier, 872 p.

8 Fletcher C.D.M., Unni K.K. \& Mertens F. 2002. World Health Organization. Pathology and genetics of tumours of soft tissue and bone. Lyon: IARC Press, 224p.

9 Gaetani P., Tancioni F., Merlo P., Villani L., Spanu G. \& Baena R.R. 1996. Spinal chondroma of the lumbar tract: case report. Surgical Neurology. 46(6): 534-539.

10 Green E.M., Adams W.M. \& Steinberg H. 1999. Malignant transformation of solitary spinal osteochondroma in two mature dogs. Veterinary Radiology \& Ultrasound. 40(6): 634-637.

11 Heblinski N. \& Schmökel H. 2017. Surgical management of myelopathy caused by a solitary spinal osteochondroma in a young cat. Journal of Feline Medicine and Surgery Open Reports. 3(1): 1-4.

12 Kahveci R., Ergüngör M.F., Günaydın A. \& Temiz A. 2012. Lumbar solitary osteochondroma presenting with cauda equina syndrome: a case report. Acta Orthopaedica et Traumatologica Turcica. 46(6): 468-472.

13 Marinho P.V.T., Jark, P.C, Avante M.L., Canola J.C., De Nardi A.B. \& Minto B.W. 2013. Tratamento cirúrgico de condroma extradural lombar em cão - relato de caso. Semina: Ciências Agrárias. 34(4): 1835-1840

14 Marinho P.V.T., Zani C.C., Jark P.C., Minto B.W. \& Costa M.T. 2014. Partial lateral corpectomy associated with pediculectomy to treat extradural chondroma in a dog: technique description. Arquivo Brasileiro de Medicina Veterinária e Zootecnia. 66(5): 1323-1328.

15 Meij B.P. \& Bergknut N. 2010. Degenerative lumbosacral stenosis in dogs. The Veterinary Clinics of North America. Small Animal Practice. 40(5): 983-1009.

16 Meuten D.J. 2016. Tumors in Domestic Animals. 5th edn. Ames: Wiley-Blackwell, 1000p.

17 Natale M., Rotondo M., D’Avanzo R. \& Scuotto A. 2013. Solitary lumbar osteochondroma presenting with spinal cord compression. BMJ Case Reports. 44 (2): 299-302.

18 Ródenas S., Pumarola M. \& Añor S. 2008. Imaging diagnosis-cervical spine chondroma in a dog. Veterinary Radiology \& Ultrasound: 49(5): 464-466.

19 Rudich S.R., Feeney D.A., Anderson K.L. \& Walter P.A. 2004. Computed tomography of masses of the brachial plexus and contributing nerve roots in dogs. Veterinary Radiology \& Ultrasound. 45(1): 46-50.

20 Sharp N.J.H. \& Wheeler S.J. 2005. Small Animal Spinal disorders. Diagnosis and Surgery. 2nd edn. Philadelphia: Elsevier Mosby, 379p.

21 Wahle A.M., Raith K., Posch B., Eddicks L., Matiasek K. \& Jurina K. 2017. Imaging diagnosis: concentric periradicular lipoma causing lumbar nerve root compression in a dog. Veterinary Radiology \& Ultrasound. 58(1): E6-E10.

22 Zaijun L., Xinhai Y., Zhipeng W., Wending H., Quan H., Zhenhua Z., Dapeng F., Jisheng Z., Wei Z. \& Jianru X. 2013. Outcome and prognosis of myelopathy and radiculopathy from osteochondroma in the mobile spine: a report on 14 patients. Journal of Spinal Disorders \& Techniques. 26(4): 194-199. 